\title{
Competencias digitales lúdicas y enseñanza
}

Graciela Esnaola Horacek. Universidad Nacional de Tres de Febrero

María Beatriz de Ansó. Universidad Nacional de Tres de Febrero
0000-0002-1436-6041

0000-0002-5826-6456

Recepción: 03.11.2019 | Aceptado: 08.11.2019

Correspondencia a través de ORCID: Graciela Esnaola Horacek

Citar: Esnaola Horacek, G. y Ansó MB. (2019). Competencias digitales lúdicas y enseñanza. REIDOCREA, 8, 399-410.

Resumen: La educación tecnológica es clave en la formación profesional docente para adquirir competencias digitales que deben ser fortalecidas desde la formación del profesorado. Desde los inicios del siglo XXI se ha incrementado la necesidad de incluir las competencias digitales en las prácticas docentes, entre ellas, experiencias con mediación de videojuegos como materiales didácticos digitales (MDD) privilegiados para la innovación pedagógica. Estas prácticas se fundamentan en nuestra línea de investigación que corrobora los beneficios de los videojuegos en aprendizajes cognitivos, emocionales, psicomotrices, además de constatar que la mayoría de los niños accede a partir de actividades videolúdicas al universo digital. En este artículo se sintetizan algunos avances obtenidos a partir del análisis de las condiciones que favorecen o dificultan buenas prácticas de enseñanza en orden a definir las características de buenas prácticas de ludificación de la enseñanza. Se expone un análisis descriptivo de enfoque cuantitativo, aplicado a un muestreo no probabilístico intencional que permite describir algunas categorías de la cultura docente en el uso de tecnología educativa y en particular de videojuegos en el aula. A partir del análisis de los datos se establecen categorías de buenas prácticas de enseñanza con videojuegos y se construyen instrumentos de autoevaluación de prácticas pedagógicas innovadoras.

Palabras clave: Tecnologías de la Información y de la Comunicación | Juego Educativo

Playful digital abilities and gamification

\begin{abstract}
Technological education is an important competence in teachers training. Technological competence involves skills for accessing and managing information sources, critical, applicative and metacognitive skills to build knowledge, capacity to formulate and resolve problems and use of educational technology. This e-competence includes actions with video-ludic mediation as a tool of teaching. The methodological framework is a quantitative approach with a descriptive scope, by means of a questionnaire validated that allows to describe some categories of the educational culture in the use of educational technology. From the analysis of the data, instruments for the self-evaluation of video-ludic pedagogical practices are built. The present work proposed to synthesize the factors that characterize the teaching practices in those environments, with the inclusion of digital games as educational resources in the classrooms and define good practices of teaching.
\end{abstract}

Keywords: Information and Communication Technologies | Educational Games

\section{Introducción}

El marco teórico de la investigación sobre la cultura docente se construyó a partir de los conceptos de buenas prácticas de enseñanza, pedagogía lúdica mediada por tecnologías de innovación, factores emocionales y juegos digitales.

Consideramos que los modelos pedagógicos mediados por tecnologías deben considerar los cambios que implican la digitalización de la palabra, del espacio y del tiempo. Si bien las migraciones de procesos analógicos a formatos digitales producen modificaciones en el soporte, en el caso de las tecnologías de la información se abre un espacio que excede el cambio tecnológico e involucra nuevos procesamientos en la estructura cognitiva y conductual (Esnaola, Ottaviano, Palacios, 2015). Esta intervención del soporte en el proceso de comunicación conlleva transformaciones profundas en los modos de aprender y en consecuencia de enseñar. 
En este escenario hemos buscado caracterizar indicios significativos en los factores que operan como facilitadores u obstáculos en las buenas prácticas de enseñanza.

Jerí Rodríguez (2008) señala respecto del concepto de Buenas Prácticas la definición del Grupo Didáctica y Multimedia (DIM) de la Universidad Autónoma de Barcelona:

[...] buenas prácticas docentes [son] las intervenciones educativas que facilitan el desarrollo de actividades de aprendizaje en las que se logran con eficiencia los objetivos formativos previstos y también otros aprendizajes de alto valor educativo, como por ejemplo una mayor incidencia en colectivos marginados, menor fracaso escolar en general, mayor profundidad en los aprendizajes. (p.40)

En ese trayecto didáctico se requiere docentes formados en competencias pedagógicas, digitales y comunicacionales (OECD, 2005) definidas como los conocimientos, destrezas y habilidades psicosociales que abarcan el uso de herramientas interactivas; interactuar en grupos heterogéneos y actuar de manera autónoma (Reyzábal, 2012).

Siguiendo los aportes del Grupo DIM, Jerí Rodríguez (2008) enumera algunos indicadores de acciones pedagógicas entendidas como buenas prácticas:

1. Generan aprendizajes significativos que se aplican en la vida diaria.

2. Los estudiantes se involucran en las actividades de aprendizaje mediante operaciones mentales, trabajo colaborativo, aprendizaje autónomo.

3. Promueven el trabajo transversal e interdisciplinar y el pensamiento divergente.

4. Incorporan el uso de las TIC y realizan procesos de evaluación permanente.

El grupo DIM también analiza los factores que favorecen buenas prácticas y enumera los siguientes:

\section{Factores relacionados con el profesorado:}

- Competencias del profesorado en la inclusión de las TIC

- Conocimiento de los recursos disponibles

- Actitud investigadora e innovadora en el aula

Factores relacionados con la gestión y administración institucional:

- El Proyecto Educativo Institucional (PEI) establece la incorporación de TIC.

- Acceso a equipamiento y recursos tecnológicos.

- Intervención de un coordinador de informática o un departamento de tecnología educativa.

- Un sistema de mantenimiento de los recursos tecnológicos.

- Estrategias de innovación favorecidas desde la gestión institucional.

- Un plan de capacitación digital, tutoría y acompañamiento a los docentes para implementar experiencias. (Marqués 2002: sep.)

De los indicadores señalados se advierte que las buenas prácticas docentes con TIC no se reducen a un conjunto de habilidades técnicas o al manejo experto de la tecnología, sino que se manifiestan en competencias curriculares y en sólida formación pedagógica. Siguiendo a Valverde (2015) señalamos que dichas prácticas integran diferentes dimensiones del proceso educativo. Lo expresa en estos términos: 
Sólo algunos docentes lo consiguen y llevan a cabo lo que se han denominado "buenas prácticas" educativas con TIC que, en definitiva, consisten en "invisibilizar" el medio y aplicar una metodología de enseñanza donde la digitalización de las herramientas de trabajo (escribir, leer, expresión artística y de representación del conocimiento) y los procesos de pensamiento (buscar, seleccionar, reproducir, analizar, valorar, sintetizar, reformular, criticar o crear) produce aprendizajes más profundos, transferibles y aplicados. ( p.14)

En esta misma línea de pensamiento, podemos destacar los aportes de Gutiérrez, Yuste, Cubo y Lucero (2011) quienes afirman:

Podemos entender como "buenas prácticas", las acciones desarrolladas en el ámbito educativo que facilitan el aprendizaje; es decir, se trata de conocer cómo hacen muchos docentes y centros junto a sus comunidades, hasta crear contextos que permitan la experimentación de nuevas fórmulas curriculares y organizativas, Yáñez (2010). Esto supone que estas prácticas deben mejorar los resultados de un proceso, responder a una experiencia sistematizada, documentada y experimentada, aplicar métodos innovadores y ser extrapolable a otros contextos. (Epper y Bates, 2004, p. 182-183).

Avanzando en esta conceptualización y, deteniéndonos en la inclusión de videojuegos en prácticas docentes hallamos que estos materiales didácticos digitales (MDD) pueden generar ambientes óptimos de aprendizaje facilitadores del desarrollo creativo, emocional y de inteligencias múltiples (Gardner, 2001) a la vez que fomentan interactividad, plantean objetivos específicos y desafíos continuos, compromiso y colaboración, ensayo y error en el aprendizaje de habilidades y una curva de aprendizaje controlado.

Los juegos digitales, los definimos como objetos de aprendizaje interactivo que se configuran en herramientas cognitivas. Incorporan componentes lúdicos, tecnológicos, artísticos, comunicativos, pedagógicos, de alto impacto. Estos objetos digitales, inmersos en ambientes de aprendizaje, se tornan en objetos que captan la motivación del estudiante precisamente por ser flexibles, deslocalizados y ubicuos, participativos y colaborativos, personales y en red.

La incorporación de videojuegos a la enseñanza traza un puente hacia su uso informal y excede al uso recreativo de ellos, aunque implican construir un ambiente ludificado de enseñanza para aprovechar al máximo su potencial. En principio, es necesario conocer las posibilidades de los videojuegos como recursos de aprendizajes y en consecuencia facilitar la accesibilidad, favorecer la usabilidad de juegos digitales de complejidad creciente y aprovechar la ubicuidad de los dispositivos móviles, además de otras plataformas, en beneficio de la jugabilidad como territorio virtual de aprendizajes.

Estudios psiconeurobiológicos reconocen, a la vez, los beneficios de experiencias inmersivas que incorporan videojuegos como recurso didáctico y favorecen aprendizajes implícitos. Algunos aportes de investigaciones pedagógicas que configuran el estado del arte de esta temática implican referenciar conceptos elaborados por algunos autores que cuentan con una trayectoria sostenida en los game studies:

- Los videojuegos son un instrumento lúdico que sirve a las nuevas generaciones, para su adaptación social en la sociedad actual (Sánchez y Peris, 1998).

- La arquitectura y metodología para el desarrollo de sistemas educativos con videojuegos merece un diseño pedagógico didáctico que lo sostenga (Gómez, 2007).

- El videojuego puede considerarse una herramienta para la pedagogía artística, la creatividad y la innovación (Escribano, 2014). 
- Los factores emocionales que aportan las narrativas merecen ser analizados en relación con su impacto en los aprendizajes (Marcano, 2014).

- Las neurociencias proporcionan fundamentos científico-fisiológicos en el estudio de la emoción, atención y estimulación que tienen su traducción en el videojuego a través de la flow experience (Crespo y Legerén, 2018).

- Los Congresos Internacionales de Videojuegos y Educación (CIVE I, II, III, IV, V, VI) celebrados desde 2012 en continuo, registran propuestas teóricas y experiencias que conjugan videojuegos y arte, salud, educación, ética e industrias culturales.

- La Asociación Alfas organizada como un grupo de investigación internacional en videojuegos y educación que tiene como objetivo registrar y difundir experiencias innovadoras en edutainment.

Para pensar la innovación en el actual escenario educativo recurrimos a Carbonell (2001) quien aporta una definición que explica:

...la innovación como una serie de intervenciones, decisiones y procesos, con cierto grado de intencionalidad y sistematización, que tratan de modificar actitudes, ideas, culturas, contenidos, modelos y prácticas pedagógicas. Y, a su vez, de introducir, en una línea renovadora, nuevos proyectos y programas, materiales curriculares, estrategias de enseñanza y aprendizaje, modelos didácticos y otra forma de organizar y gestionar el currículum, el centro y la dinámica del aula. (p.4)

La innovación educativa en la era digital reconoce como tendencia clave a la ludificación de los procesos de enseñanza. Ampliando el concepto sostenemos que ludificar implica incorporar videojuegos o dinámicas de juego con intencionalidad educativa en entornos de aprendizaje que se diseñan en el círculo mágico del juego que propone Campbell (1959). Cruzar el umbral mágico implica un tránsito hacia una esfera de renacimiento que aparece simbolizada en todas las culturas. De allí la potencia de los entornos narrativos que sumergen a los participantes en la identificación con el camino del héroe citado por Campbell.

Si comprendemos la riqueza narrativa y simbólica que implican estos relatos lúdicos se diseñará un modelo de enseñanza-aprendizaje que enfatiza el compromiso, el aprendizaje en la práctica, la colaboración, la reflexión, la repetición, la retroalimentación frecuente y el compartir. Las actividades de aprendizaje se estructuran entonces, en torno a los retos de la vida real o de ficción que obligan a los alumnos a tomar una variedad de roles a medida que se identifican de manera activa y buscan las herramientas y la información multidisciplinar que necesitan para generar soluciones. También, está asociado directamente al conocido como aprendizaje basado en los retos o en problemas. (Revuelta, 2014)

Profundizando en la descripción, es interesante proponer la construcción de una pedagogía lúdica incidental que implica el diseño de un entorno de enseñanza ludificado que se sostenga en comunidades de aprendizaje que proponen prácticas colaborativas de aprendizaje y enseñanza, tal como sucede en el ámbito del juego social. De esta manera, esta pedagogía no explícita generará en los participantes las llamadas "experiencias flow - experiencias óptimas" potenciadoras del pensamiento creativo. Asimismo, los entornos personales de aprendizaje lúdico consideran la movilidad y la interacción entre los participantes, la sensibilidad al contexto en el entorno del usuario y la transparencia de los mecanismos pedagógicos. De este modo, la pedagogía incidental, no explícita, es mucho más potente dado que vincula las emociones y la inmersión participativa sin estar supeditado al control curricular. 
Los juegos digitales son los objetos lúdicos paradigmáticos de la cultura contemporánea. Son objetos culturales hipermediales de potencial educativo, que han logrado penetrar y colonizar expresiones multiculturales diversas. Han modificado las formas de percepción de tiempo-espacio y han transformado también la construcción de subjetividad desde lo personal y las formas de interacción humana y laboral, desde lo social. Los videojuegos, que consideramos un nuevo "hipergénero artístico emergente" de la cultura contemporánea se inscribe en la cultura digital que prioriza la mixtura, la convergencia de géneros y estilos en un producto innovador.

En consecuencia, las buenas prácticas de enseñanza con videojuegos como recurso didáctico crean un espacio renovador en los modelos tradicionales de enseñanza. Los videojuegos son recursos didácticos potentes en la inclusión de TIC en el aula, y su inclusión en la enseñanza favorece el desarrollo de la creatividad, el pensamiento estratégico y el aprendizaje colaborativo. El modelo de enseñanza basado en juegos digitales incentiva la motivación, la participación y el desarrollo de competencias cognitivas, comunicacionales, emocionales y tecnológicas (Sampedro Requena y Mcmullin, 2015).

En especial los factores emocionales son estructurantes de experiencias óptimas. Marcano (2014) explica que motivación y emoción han sido abordadas desde perspectivas distintas y separadas, pero están íntimamente relacionadas. La motivación, que impulsa la conducta, está impregnada de emociones y las emociones motivan la conducta. Los videojuegos provocan emociones y constituyen un medio controlado de interacción social y emocional -interés, felicidad, humor, sorpresa, ansiedad, hostilidad, asco, ira, tristeza y amor-.

La inclusión de videojuegos en prácticas docentes puede generar ambientes óptimos de aprendizaje facilitadores del desarrollo creativo, emocional y de inteligencias múltiples (Gardner, 2001) a la vez que fomentan interactividad, plantean objetivos específicos y desafíos continuos, compromiso y colaboración, ensayo y error en el aprendizaje de habilidades y una curva de aprendizaje controlado.

\section{Objetivos o hipótesis}

El objetivo general de este artículo es caracterizar y analizar buenas prácticas de enseñanza mediadas por juegos digitales como recursos educativos. Son objetivos específicos:

1. Analizar las condiciones que favorecen buenas prácticas mediadas por videojuegos en la enseñanza de disciplinas curriculares.

2. Indagar los obstáculos epistemológicos y epistemofílicos que dificultan la incorporación de videojuegos en el aula.

3. Diseñar un instrumento de recogida de información de buenas prácticas de enseñanza con videojuegos.

\section{Métodos}

La inclusión de tecnologías digitales en procesos de aprendizaje delimita el escenario sociocultural del problema a investigar y diseñar un proyecto de interés pedagógico y viabilidad fáctica. Específicamente se ha detectado que la cultura docente no reconoce los saberes que se construyen en espacios informales estableciendo una frontera entre la vida cotidiana de los estudiantes y la vida escolar. Este es el primer obstáculo para resolver haciendo visible la impronta de la cultura lúdica en los estilos de aprendizaje. 
Siguiendo a Hernández Sampieri (2006), los criterios que justifican nuestra indagación son:

- Conveniencia: Los videojuegos son dispositivos potentes que mediatizan aprendizajes de competencias complejas, y pueden ser importantes aliados de actividades de enseñanza.

- Relevancia social: Creemos que los resultados de esta investigación fortalecen saberes que benefician a ambos actores del proceso educativo: a los docentes les provee de herramientas teóricas y tecnológicas de alto impacto para la enseñanza y a los alumnos les permite acceder al aprendizaje legitimando un medio de entretenimiento valorado.

- Implicaciones prácticas: la inclusión de juegos digitales en el aula puede generar mejoras en los procesos de aprendizaje, y difusión e implementación de pedagogías videolúdicas, favoreciendo la motivación, el desarrollo de competencias cognitivas, emocionales, comunicacionales, tecnológicas.

- Valor teórico: Creemos que es relevante la necesidad de incorporar o acrecentar en los Institutos Superiores de Formación Docente y Profesorados Universitarios conocimiento teórico práctico en Tecnología Educativa y modelos de innovación que incluyan pedagogías videolúdicas.

- Utilidad metodológica: Consideramos que esta investigación aporta datos significativos validados para enriquecer modelos pedagógicos ludificados que favorezcan los aprendizajes en la actual sociedad digital. Esto es pensar la gamificación como tendencia clave en entornos educativos (Informe Horizon 2014).

El enfoque metodológico seleccionado ha sido cuantitativo de alcance descriptivo de modo tal que permita delinear una caracterización de la cultura docente con relación al objeto de estudio.

\section{Instrumento}

El instrumento que se diseñó a los efectos de este estudio fue un cuestionario que permitió describir aspectos de la cultura docente en el uso de videojuegos en el aula. El cuestionario ha sido validado internacionalmente según criterios de univocidad, pertinencia e importancia y se aplicó en los años 2016 y 2017.

El cuestionario se estructuró en 3 dimensiones:

1. Datos personales y socioeducativos: Sexo, edad, nivel de instrucción, antigüedad, nivel educativo, cargo, área de formación, gestión estatal o privada.

2. Experiencia con videojuegos: Juega videojuegos, cuánto, opinión sobre aspectos positivos y conductas negativas.

3. Videojuegos y educación: Capacitación en Tecnología educativa, recursos que utiliza, incluyó videojuegos, con qué función, tuvo dificultades, cuáles, cómo resultaron las evaluaciones, que conductas favorecen los videojuegos.

\section{Participantes}

El cuestionario se aplicó a un muestreo no probabilístico operático o intencional (Ferrer, 2010). Y la población del estudio se compuso de 152 docentes de distintas disciplinas y niveles del sistema educativo en actividad, en la provincia de Buenos Aires, Argentina. 


\section{Resultados}

Los datos personales y socioeducativos registrados en la Dimensión 1 del Cuestionario señala que se han encuestado 152 docentes, de los cuales 113 (74.3\%) son mujeres. Esto representa casi tres cuartas partes de mujeres, frente a un cuarto de varones. La disparidad entre hombres y mujeres es un dato relevante en cuanto a los porcentajes que, según el sexo, eligen la profesión docente, y que tiende a mantenerse constante en diversos estudios en Argentina. El Censo Nacional de Personal de los Establecimientos Educativos realizado por el Ministerio de Educación de la Nación, afirma que la cantidad de docentes en el sistema educativo argentino aumentó un $28,6 \%$ entre 2004 y 2014 , de los cuales tres de cada cuatro (el $75,7 \%$ ) son mujeres, en su mayoría tienen formación y son consumidores de productos culturales (CeNPE, 2014). este dato, en perspectiva de género, se cruza con la tradición de que son los varones los usuarios más frecuentes de tecnologías y en particular, videojuegos. Este aspecto está siendo cuestionado incorporando a mujeres en la industria, como desarrolladoras o en los juegos diseñados con perspectiva de género, apartándose de las primeras narrativas, más vinculadas a shooters, carreras de autos o juegos de escenarios militares.

En el estudio que nos ocupa las edades de los encuestados oscilan entre 20 y 60 años con una edad media de 41.67 años y un desvío standard de 10.28 años. Más de un tercio de la población se encuentra entre los 40 y los 49 años. $Y$ un cuarto del total pertenece al sector de más de 50 años, lo cual sumado al grupo más elevado en porcentaje resulta que más del $50 \%$ de la población total tiene una edad media-alta y transita la mitad o más de su carrera profesional. Esto resulta ser indicio de una actitud favorecedora ante los videojuegos, si han sido incorporados a los juegos de dicha generación o si no pertenecían a su universo lúdico, con esa limitación de época.

En relación con la formación, se ha observado que el máximo nivel de instrucción alcanzado por el $67.7 \%$ de los docentes encuestados es superior universitario, completo $(42,27 \%)$ e incompleto $(61,40.1 \%)$. Los datos expresan la decisión manifiesta de más del $55 \%$ de los docentes consultados de alcanzar niveles superiores de formación a través de diferentes propuestas, cursos, carreras de complementación docente, carreras de grado o posgrado.

La antigüedad en la docencia se encuentra polarizada, a saber, el $50 \%$ del total encuestado tiene una antigüedad entre uno y diez años y el $34,9 \%$ del total con antigüedad superior a 15 años, lo cual coincide con el porcentaje que arroja la población entre 40 y 49 años.

De estos docentes, el $62,5 \%$ se desempeña en el nivel secundario y el $34,9 \%$ lo hace en el nivel primario, los restantes reparten sus dedicaciones en los niveles inicial, universitario y no universitario, y casi el $40 \%$ ejerce en distintos niveles simultáneamente.

De los encuestados, el $67,8 \%$ ejercen funciones docentes, el resto se distribuye en forma bastante equitativa entre cargos de gestión, asesoría, técnicos y administrativos.

El grupo más numeroso de docentes - el 75\%- ha sido formado en las áreas de sociales y técnicas. Observamos la disposición mayoritaria de los docentes de estas áreas a capacitarse en el uso educativo de las TIC. La formación en otras áreas es minoritaria. 
De este conjunto de docentes el $31,6 \%$ lo hace en forma exclusiva en instituciones de gestión privada mientras que el $50 \%$ se desempeña exclusivamente en instituciones estatales, el resto comparte sus actividades en ambos tipos de establecimientos.

Tabla 1. Dimensión 1: Datos personales y socioeducativos.

\begin{tabular}{|c|c|}
\hline & \multicolumn{1}{c|}{$\begin{array}{c}\text { MUESTRA } \\
\text { Dimensión I } \\
\text { Datos personales y socio-educativos }\end{array}$} \\
\hline & $\begin{array}{l}\text { Docentes que cursan licenciaturas en Untref y capacitaciones del Programa Nuestra } \\
\text { Escuela. }\end{array}$ \\
\hline $\mathbf{7 4 \%}$ & mujeres, entre 20 y 60 años, con una edad media de 41 años. \\
\hline $\mathbf{M a ́ s}$ del 50\% & transita la mitad o más de su carrera profesional. \\
\hline $\mathbf{6 7 \%}$ & educación superior universitaria. \\
\hline $\mathbf{5 0} \%$ & se desempeña en instituciones estatales \\
\hline $\mathbf{6 2} \%$ & trabaja en el nivel secundario. \\
\hline $\mathbf{7 5 \%}$ & ha sido formado en áreas sociales y técnicas. \\
\hline
\end{tabular}

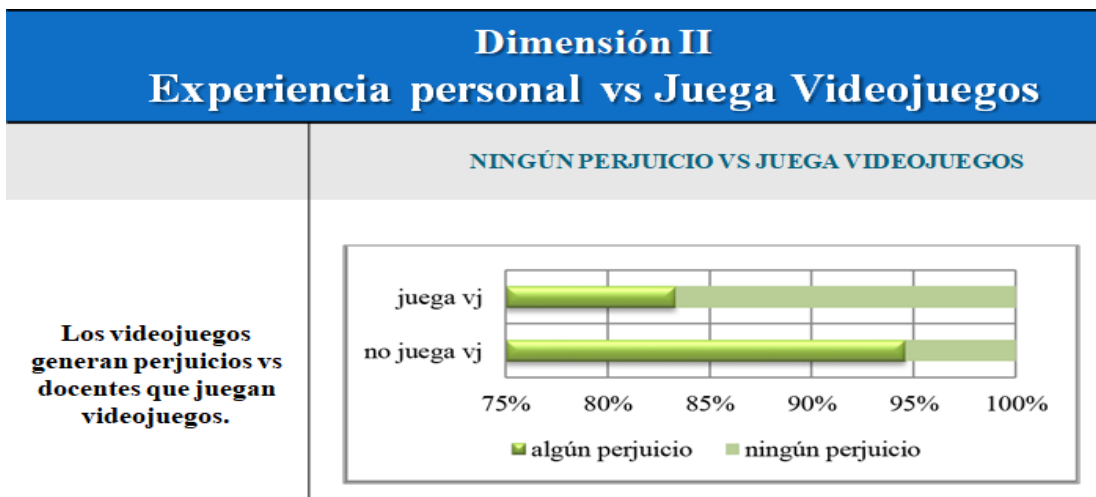

Figura 1: Dimensión 2: Experiencia con videojuegos.

En la dimensión 3, vinculada a la capacitación en TIC se verifica que gran parte de los docentes encuestados han recibido poca, insuficiente o ninguna capacitación digital durante su formación e incluso durante su trayectoria profesional.

\section{Dimensión III \\ Capacitación digital en etapa de formación}

62.8\% recibió poca, insuficiente o ninguna capacitación digital en su etapa de formación profesional.

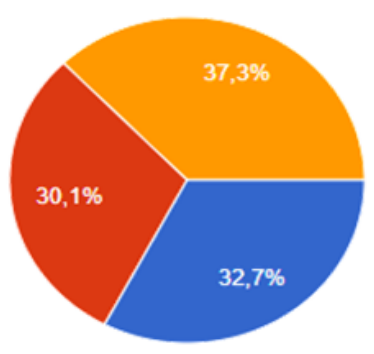

No recibi ninguna capacitación digital

Recibi poca/insuficiente capacitación digital

Sí, recibí importante/adecuada capacitación digital

Figura 2: Dimensión 3. Videojuegos y Educación. 
Relacionando la dimensión 2 y 3 en términos de formación en Tecnología educativa vs tiempo de juego se aprecia que con el aumento de capacitación en Tecnología educativa aumenta la frecuencia de juego.

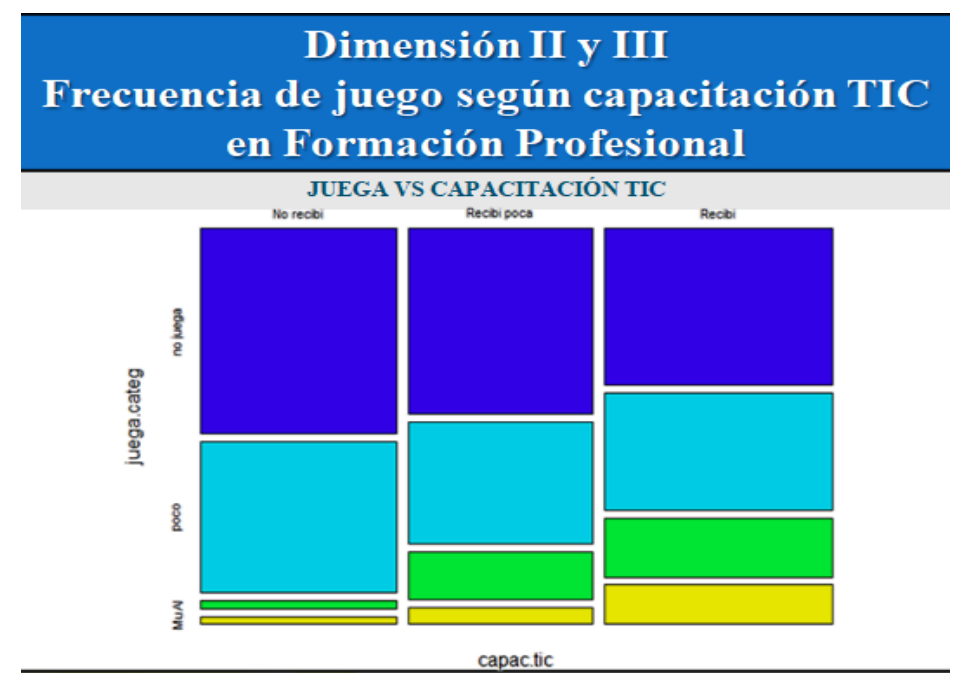

Figura 3: Dimensión 2 y 3. Capacitación vs juega videojuegos.

Al respecto, se encontró asociación estadística significativa entre la práctica videolúdica del docente y los resultados de los alumnos en las evaluaciones de aprendizajes mediados por videojuegos, en proporción significativamente mayor de exámenes con calificaciones bueno o muy bueno entre los que juegan videojuegos y los que no lo hacen.

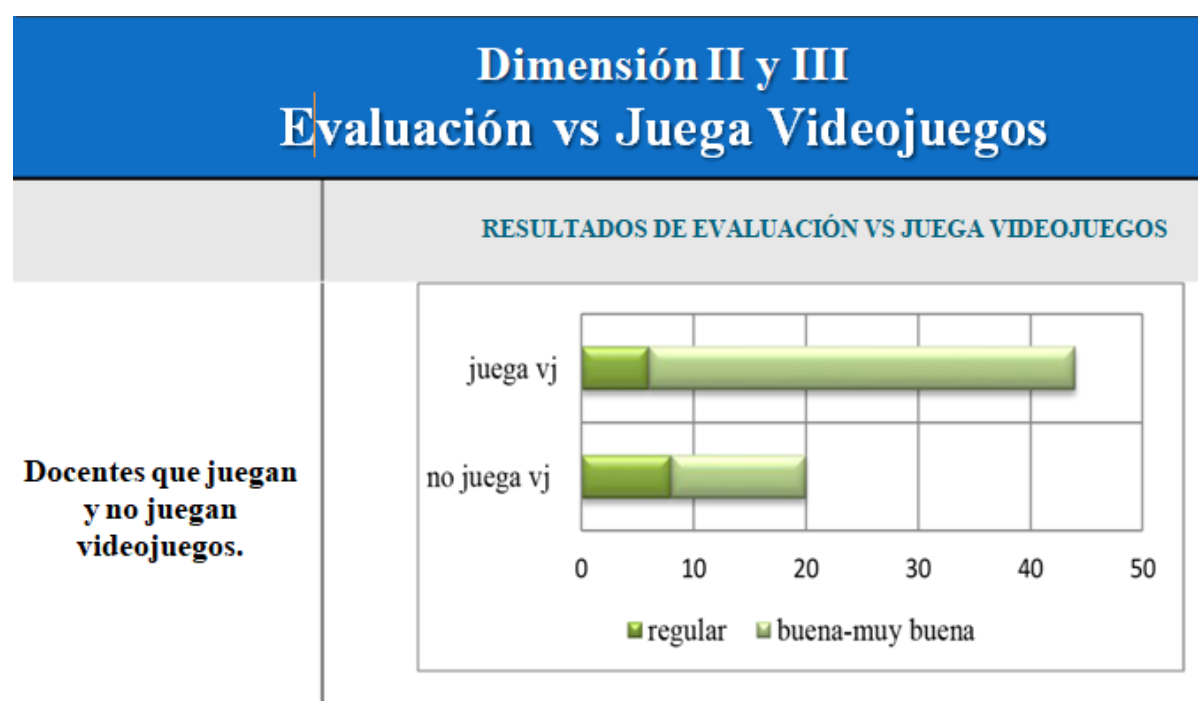

Figura 4. Dimensión 2 y 3 . Evaluación vs juega videojuegos.

En un análisis de correspondencias múltiples entre docentes que juegan videojuegos o no, la función evaluación y los beneficios, se identificaron dos grupos. Los docentes que juegan videojuegos y los usan para evaluación refieren beneficios de los videojuegos en relación con la toma de decisiones, el pensamiento anticipatorio, la reflexión crítica y la inteligencia emocional. Por el contrario, los docentes que no juegan videojuegos a la vez no los usan con función de evaluación ni reconocen sus beneficios. 
Otro aspecto para destacar en el análisis de los datos permitió esclarecer las siguientes dimensiones de buenas prácticas de enseñanza con videojuegos:

- Dimensión cognitiva: De acuerdo con los resultados del cuestionario, asociar recursos videolúdicos a prácticas educativas favorece el desarrollo cognitivo. Se encontraron diferencias significativas entre las proporciones a favor del beneficio de jugar sobre la reflexión crítica, la toma de decisiones y el pensamiento estratégico y anticipatorio de parte de los docentes que juegan videojuegos con relación a las opiniones de quienes no juegan. Con porcentajes superiores al $50 \%$ los docentes afirman que los videojuegos favorecen el desarrollo cognitivo, el autoaprendizaje y la creatividad. Estas categorías dan cuenta de lo que se denomina "función enseñante de los objetos tecnológicos".

- Dimensión didáctica: La ludificación es una práctica de innovación que requiere competencias lúdicas. Estas categorías surgen claramente en el análisis de las condiciones que favorecen buenas prácticas de enseñanza con videojuegos. El primer dato significativo resultó ser el elevado porcentaje de docentes que no juegan videojuegos. Aun así, el $98 \%$ reconoce beneficios o aspectos positivos de jugarlos. Aunque también en alto porcentaje (88\%) afirman que la adicción y la pérdida de tiempo de estudio son consecuencias negativas de video jugar. En referencia a las competencias lúdicas es de destacar que no se encontraron diferencias significativas en la distribución de esta variable para las distintas categorías de sexo y edad. Se destaca también que la innovación no es sólo resultado de decisiones curriculares individuales, sino que debe ser fruto de políticas públicas y de decisiones institucionales o de centro.

- Dimensión emocional: En esta dimensión se destaca la motivación como un factor condicionante de gran relevancia al evaluar la actitud de predisposición de los alumnos al aprendizaje. El entretenimiento es el beneficio más reconocido, asociado naturalmente a la motivación. Y a la vez la motivación se registra, por parte de los docentes, como una función de uso de videojuegos en el aula para rentabilizar el interés que despiertan al servicio del aprendizaje. Los docentes refieren la importancia del aprendizaje colaborativo en cuanto aspecto positivo que es consecuencia de jugar con otros.

Se encontró diferencia significativa entre las opiniones a favor del beneficio de jugar videojuegos respecto del desarrollo de la inteligencia emocional por parte de quienes juegan en relación con los docentes que no juegan. A fin de contrarrestar los potenciales riesgos de dichas actividades, la educación formal es responsable de promover el análisis crítico y el fortalecimiento de valores:

Debemos tener en cuenta la responsabilidad de los educadores (escuela y familia) de ofrecer herramientas educativas, instrumentos de análisis crítico que permitan una reflexión de los contenidos ofrecidos por los videojuegos, pues éstos son una fuente de asentamiento de valores y potenciación de actitudes y formas de resolver conflictos. No cabe duda de que el ámbito escolar es un espacio desde el que tenemos que intervenir para conseguir una sociedad sin discriminaciones, y avanzar en la igualdad de género. (Sahuquillo Mateo, Ros Ros, Bellver Moreno, 2008, p. 146)

- Dimensión tecnológica: más del $30 \%$ de los encuestados no recibieron capacitación en TIC durante su etapa de formación. Y en porcentaje similar tampoco recibieron capacitación en tecnología educativa durante su trayectoria profesional. El $50 \%$ de los docentes refiere haber recibido capacitación voluntaria. Pero cuando se pregunta específicamente por videojuegos el $75 \%$ responde haber incluido pocos o ninguno como herramienta didáctica. Los docentes que incluyen juegos digitales expresan diferentes funciones de uso, 
entre ellas el entrenamiento de habilidades procedimentales, la experimentación y la búsqueda de información. Respecto de las dificultades se encontró asociación estadística significativa sobre la carencia de recursos. En el caso de quienes recibieron capacitación en TIC en su etapa de formación las diferencias fueron registradas en las dificultades de conexión y carencia de recursos.

Con los resultados del Estudio y el análisis de los datos obtenidos, se configuraron las características de buenas prácticas de enseñanza con juegos digitales y se elaboró una Ficha de autoevaluación (en base a la ficha de Valverde de autoevaluación de Buenas Prácticas educativas con TIC). Se fortalecieron con evidencias empíricas las siguientes dimensiones: Cognitiva, Organizativa, Didáctica, Emocional y Tecnológica con sus categorías y 4 niveles de evaluación.

\section{Discusión}

Al finalizar nuestra indagación corroboramos la hipótesis inicial: la cultura docente no reconoce los saberes que se construyen desde los videojuegos. Esta afirmación contrasta con las tendencias emergentes que establece el Informe Horizon 2017 y que plantea como desafío solucionable la integración del aprendizaje formal e informal, y, en consecuencia, la legitimación de aprendizajes multicontextuales en el ámbito de la educación formal.

Conforme a los objetivos planteados para el estudio que hemos descripto en este artículo concluimos que:

- Se caracterizaron aspectos de la cultura docente, señalando condiciones que favorecen o dificultan el uso de videojuegos en el aula y se determinaron dimensiones de buenas prácticas de enseñanza con juegos digitales.

- Se aplicaron criterios que definen las buenas prácticas de enseñanza con TIC, entendidas como buenas prácticas de enseñanza con videojuegos que refieren al conjunto de acciones pedagógicas (diagnóstico, planificación, ejecución, control y evaluación del proceso educativo) que incorporan a los juegos digitales como recursos educativos y logran resultados satisfactorios de aprendizaje.

- Los docentes poseen insuficiente formación en tecnología educativa para producir innovación pedagógica. El uso de videojuegos requiere competencias pedagógicas y tecnológicas que permitan otorgarles significatividad como mediadores de aprendizajes. La formación del profesorado debe profundizar la consolidación de los fundamentos teóricos de las pedagogías lúdicas y la sistematización de los principios didácticos de buenas prácticas de enseñanza con videojuegos.

- El docente que tiene experiencia con juegos digitales, esto es, que jugó o usó videojuegos, tiene una significativa predisposición para implementar actividades con mediación video lúdica.

- Los docentes reconocen los saberes adquiridos con los videojuegos por los alumnos. Sin embargo, no incorporan los saberes previos de los alumnos en sus prácticas de aula.

- Como producto de este estudio se ha logrado diseñar una Ficha de autoevaluación de buenas prácticas con videojuegos.

En relación con las limitaciones del estudio, se puede mencionar que no se logró describir la cultura lúdica de los docentes, con el instrumento diseñado. Se debería implementar una indagación de corte cualitativo para lograr más información al respecto. Otra limitación fue la dificultad de acceder a los colegios para realizar un seguimiento cercano a las prácticas docentes, poder estudiar la implicancia de los equipos directivos 
en las experiencias de innovación con videojuegos y corroborar en las aulas los proyectos de ludificación.

Del trabajo realizado podemos señalar algunas líneas de investigación futura vinculadas al diseño de un modelo pedagógico de buenas prácticas de enseñanza con juegos digitales y a definir las competencias que requiere la Formación Docente en el área de la Pedagogía Lúdica. Otra línea de interés a ser desarrollada se relaciona con el consenso y validación de criterios de evaluación pedagógica de videojuegos.

\section{Referencias}

Asociación Grupo Alfas (2017). Congresos CIVE y Proyectos.

Campbell, J (1959). El héroe de las mil caras. Psicoanálisis del mito. México D.F.: Fondo de Cultura Económica.

Carbonell, J. (2001). La innovación educativa hoy. En J. Carbonell, La aventura de innovar. El cambio en la escuela. Madrid, España: Ediciones Morata.

Crespo, V. y Legerén, B. (2018). El uso de la Neurociencia en el diseño de contenidos transmedia en los canales de televisión públicos de Europa: Videojuegos y Social TV. En Revista Edmetic. 7 (2), 46-57.

Escribano, F. (2014). El videojuego como herramienta para la pedagogía artística. Creatividad e innovación (Tesis Doctoral). Universidad Complutense de Madrid, España.

Esnaola, G., Ottaviano, M. y Palacios, M. (2015). Migraciones, digitalizaciones y prácticas educativas en el contexto argentino. Educatio Siglo XXI, 33 (3), 63-84.

Ferrer, J. (2010). Metodología de la Investigación: Tipos de muestreo [Artículo de Blog]

Gardner, H. (2001). Estructuras de la Mente. La Teoría de las Inteligencias Múltiples. México, D.F.: Fondo de Cultura Económica.

Gómez, M. (2007) Arquitectura y metodología para el desarrollo de sistemas educativos basados en videojuegos (Tesis Doctoral). Universidad Complutense de Madrid.

Gutiérrez Esteban, P; Yuste Tosina, R; Cubo Delgado, S; Lucero Fustes, M. (2011). Buenas prácticas en el desarrollo de trabajo colaborativo en materias TIC aplicadas a la educación. Profesorado Revista de Currículum y formación del profesorado, 15(1), 24-36.

Hernández Sampieri, R., et al. (2006). Metodología de la investigación. México: Mc Graw Hill (4ta edición).

Instituto Nacional de Tecnologías Educativas y de Formación del Profesorado (INTEF) (2017). Resumen Informe Horizon Edición 2017 Educación Superior.

Jerí Rodríguez, D. (2008). Buenas prácticas en el ámbito educativo y su orientación a la gestión del conocimiento. Revista Educación, PUCP, 17 (33), 29-48.
Johnson, L., Adams Becker, S., Estrada, V. y Freeman, A. (2014). Informe Horizon 2014 Edición de educación Superior. Austin, Texas: The New Media Consortium.

Marcano, B. (2014). Factores emocionales en el diseño y la ejecución de videojuegos y su valor formativo en la sociedad digital. El caso de los videojuegos bélicos (Tesis Doctoral). Universidad de Salamanca.

Ministerio de Educación y Deportes (2014). CENPE, Censo Nacional del Personal de los Establecimientos Educativos. Datos generales. Buenos Aires: Ministerio de Educación y Deportes.

OECD. (2005). The definition and Selection of key Competencies. Executive Summary.

Reyzábal, M. (2012) Las competencias comunicativas y lingüísticas, clave para la calidad educativa. En REICE Revista Iberoamericana sobre Calidad, Eficacia y Cambio en Educación, 10(4), 64-77.

Revuelta Domínguez, F. (2014). "Aprendizaje informal y formal en la metodología Game Based Learning: modelo de intervención didáctica y evaluación de competencias". Conferencia magistral en las $\mathrm{V}$ Jornadas pedagógicas en tecnología e innovación educativa. Universidad Casa Grande, Guayaquil.

Sahuquillo, P., Ros Ros, C. y Bellver Moreno, C. (2008). El rol de género en los videojuegos. En Revista electrónica Teoría de la Educación. 9 (3), 24-31.

Sampedro Requena, BE. y Mcmullin, KJ. (2015). Videojuegos para la inclusión educativa. Digital Education Review, 27, 122-137.

Sánchez i Peris, F. (1998). Valores, estilos de vida y educación de los niños en relación al videojuego: bases para un programa de acción. (Tesis Doctoral). Universitat de València, España.

Valverde Berrocoso, J. (2015). El Proyecto de educación digital en un centro educativo. Madrid: Editorial Síntesis SA.

Valverde Berrocoso, J. (2011). Docentes E-Competentes. Buenas Prácticas con TIC. Barcelona: Octaedro S.L. 